\title{
THE DYNAMICS OF MAPS OF SOLENOIDS HOMOTOPIC TO THE IDENTITY
}

\author{
ALEX CLARK
}

\begin{abstract}
Given a map of a solenoid homotopic to the identity, we use its difference from the identity to study its rotation and the nature of its fixed point set. With the aid of hyperspace theory, we show that a certain class of solenoids admits no expansive homeormorphism.
\end{abstract}

\section{INTRODUCTION}

Corresponding to a given sequence of natural numbers $N=\left(n_{1}, n_{2}, \ldots\right)$ with $n_{j} \geq 2$ for each $j$ there is the solenoid $\Sigma_{N}$, the inverse limit

$$
S^{1} \stackrel{n_{1}}{\longleftarrow} S^{1} \stackrel{n_{2}}{\longleftarrow} S^{1} \stackrel{n_{3}}{\longleftarrow} \cdots \quad \Sigma_{N}=\left\{\left\langle z_{j}\right\rangle_{j=1}^{\infty} \in \prod_{i=1}^{\infty} S^{1} \mid z_{j}=z_{j+1}^{n_{j}} \text { for } j=1,2, \ldots\right\} .
$$

If $\prod_{i=1}^{\infty} S^{1}$ is given the group operation (denoted "+ " ) of component-wise multiplication, then each solenoid $\Sigma_{N}$ is a topological subgroup. Letting $\rho$ denote the metric on $S^{1}$ inherited from the Euclidean metric on $\mathbb{R}^{2}$, we obtain a translation invariant metric $d$ for $\prod_{i=1}^{\infty} S^{1}$ and hence $\Sigma_{N}$

$$
d\left(\left\langle x_{j}\right\rangle_{j=1}^{\infty},\left\langle y_{j}\right\rangle_{j=1}^{\infty}\right)=\sum_{j=1}^{\infty} \frac{1}{2^{j}} \rho\left(x_{j}, y_{j}\right) .
$$

Also, each solenoid $\Sigma_{N}$ admits a fibration with unique path lifting $\pi_{N}: \mathbb{R} \rightarrow \Sigma_{N}$ onto the arc component of the identity $e=(1,1, \ldots)$ given by

$$
t \mapsto\left\langle\exp (2 \pi i t), \exp \left(\frac{2 \pi i t}{n_{1}}\right), \ldots, \exp \left(\frac{2 \pi i t}{n_{1} \cdots n_{j}}\right), \ldots\right\rangle,[\mathrm{C} 1] .
$$

Given any map $f: \Sigma_{N} \rightarrow \Sigma_{N}$ homotopic to the identity, the map $\delta(x)=f(x)-x$ is homotopic to the map with constant value $e$ and so is lifted by a map $\widetilde{\delta}$ making the following diagram commute

$$
\begin{aligned}
& \mathbb{R} \\
& \widetilde{\delta} \nearrow \downarrow \pi_{N} . \\
& \Sigma_{N} \underset{\delta}{\longrightarrow} \Sigma_{N}
\end{aligned}
$$

A character of $\Sigma_{N}$ is a continuous homomorphism $\chi: \Sigma_{N} \rightarrow S^{1} \subset \mathbb{C}$. Any continuous map $\Sigma_{N} \rightarrow \mathbb{C}$ may be uniformly approximated by linear combinations of finitely many characters of $\Sigma_{N}$, see, e.g., [P],[Wa]. Thus for $x \in \Sigma_{N}$ the induced map $\Delta_{x}: \mathbb{R} \rightarrow \mathbb{R}, t \mapsto \widetilde{\delta} \circ\left(x+\pi_{N}(t)\right)$ is a limit periodic function, meaning that it can be uniformly approximated to within any prescribed $\varepsilon>0$ by a periodic

Date: May 27, 2000.

This work was funded in part by a faculty research grant from the University of North Texas. 
function (depending on $\varepsilon$ ). In our case we may use trigonometirc polynomials with rational periods for these approximations, (see, e.g., [C2]). In what follows we will use the map $\widetilde{\delta}$ to describe the dynamics of $f$.

Since any homeomorphism of $\Sigma_{N}$ which does not permute its composants is homotopic to the identity (see [F] 1 Theorem 2.6) and since $\Sigma_{N}$ admits a minimal almost periodic flow which has its composants as orbits, it follows from Theorem 3.8 of $[\mathrm{CHM}]$ that any closed subset of $\Sigma_{N}$ may be realized as the fixed point set of a homeomorphism of $\Sigma_{N}$ homotopic to the identity. Our analysis will show, however, that any isolated continuum of fixed points is necessarily unstable under perturbations of the map. In fact, this result generalizes to maps homotopic to the identity map of any one-dimensional compact space (other than $S^{1}$ ) that admits a minimal flow.

The structure of the group of homeomorphisms of the solenoid $\Sigma_{N}$ has been described topologically in $[\mathrm{K}]$ and is known to depend crucially on $N$. Independent of $N$ there is always the subgroup of homeomorphisms $E_{N}$ consisting of all homeomorphisms which are homotopic to a composition of one or more of the following: the involution $x \mapsto-x$ and a (possibly trivial) translation. We shall show that no homeomorphism from $E_{N}$ can be expansive. For a certain class of solenoids, $E_{N}$ is the entire group of homeomorphisms, and so no solenoid from this class admits an expansive homeomorphism. Hence, while the dyadic solenoid is one of the primary examples of a hyperbolic invariant set, there is an entire class of solenoids no member of which can occur as a hyperbolic invariant set, see, e.g, [Rob].

\section{Rotation Number}

As in [C2], with $p_{j}: \Sigma_{N} \rightarrow S^{1}$ denoting the projection onto the $j^{\text {th }}$ coordinate and $\widehat{N}$ denoting the subgroup of $(\mathbb{Q},+)$ generated by

$$
\left\{\frac{1}{n_{1}}, \frac{1}{n_{1} n_{2}}, \ldots, \frac{1}{n_{1} n_{2} \cdots n_{j}}, \ldots \mid j=1,2, \ldots\right\}
$$

$X(N)=\left\{\chi_{r}: \Sigma_{N} \rightarrow S^{1} \mid r \in \widehat{N}\right\}$ is the group of characters of $\Sigma_{N}$, where for

$$
r=\frac{m}{n_{1} n_{2} \cdots n_{j}} \in \widehat{N}, \chi_{r}(x)=\left(p_{j+1}(x)\right)^{m} .
$$

Given a map $f: \Sigma_{N} \rightarrow \Sigma_{N}$ homotopic to the identity, the associated map $\widetilde{\delta}$ has an expansion

$$
\widetilde{\delta}(x) \sim \delta_{0}+\sum_{r \in \widehat{N}-\{0\}} \delta_{r} \chi_{r}(x) .
$$

The constant term

$$
\delta_{0} \text { is } \int_{\Sigma_{N}} \widetilde{\delta}(x) d \mu_{N},
$$

where $\mu_{N}$ is the Haar measure, which is the unique invariant normalized measure of the strictly ergodic almost periodic standard flow

$$
(t, x) \mapsto \pi_{N}(t)+x
$$

(see, $[\mathrm{NS}] \mathrm{V}, 9.34, \mathrm{p} .510)$. Hence, $\delta_{0}$ is also the mean value of each function $\Delta_{x}$ :

$$
\delta_{0}=\lim _{T \rightarrow \infty} \frac{1}{T} \int_{0}^{T} \Delta_{x}(t) d t .
$$


Hence, $\delta_{0}$ provides a measure of the average amount a point is moved along its composant by the map $f$. In general, this simple-minded rotation number is not as telling as the rotation number of an orientation-preserving homeomorphism of $S^{1}$, but the following general result does hold.

Theorem 2.1. If $\delta_{0}=0$, then the associated map $f$ has uncountably many fixed points.

Proof. It is easy to construct functions with mean value 0 which do not take on the value 0 , but we shall show that this is not possible for limit periodic maps. After doing so, it follows that each function $\Delta_{x}$ has at least one zero. Since $\Sigma_{N}$ has uncountably many composants, this yields the desired result.

Suppose then that $g: \mathbb{R} \rightarrow \mathbb{R}$ is limit periodic with mean value 0 and that $g$ is never 0 . By continuity we may assume without loss of generality that $g$ is positive. Let $m=\min \{g(t) \mid t \in[0,1]\}>0$ and let $P \geq 1$ be a period of a periodic function which uniformly approximates $g$ to within $m / 2$. Then on each interval $I_{n}=[(n-1) P, n P], n=1,2, \ldots$ we have $\int_{I_{n}} g(t) d t \geq m / 2$. For large enough $n$,

$$
\frac{1}{n P} \int_{0}^{n P} g(t) d t \geq \frac{m}{2 P}
$$

will be within $\frac{m}{4 P}$ of the mean value of $g$. This contradicts $g$ having mean value 0 . In fact, we can conclude that if $g$ is not identically 0 it must have both positive and negative values.

\section{Fixed Point Sets}

While every closed subset of $\Sigma_{N}$ occurs as the fixed point set of some homeomorphism $\Sigma_{N} \rightarrow \Sigma_{N}$ homotopic to $i d_{\Sigma_{N}}$ [CHM], the most significant fixed point sets dynamically are those which persist under small perturbations of $f$. Interestingly, the topological structure of the set of fixed points is sufficient to determine that certain fixed points disappear under arbitrarily small perturbations. In particular, it will be shown that isolated fixed points are unstable under perturbations of the map. In fact, the theorem applies to any one-dimensional compact metric space $M \neq S^{1}$ which is the minimal set of a flow $\phi_{M}: \mathbb{R} \times M \rightarrow M$. Any such space $M$ is an example of an orientable matchbox manifold (see [AM]). In what follows, we consider a map $f^{\prime}$ to be a perturbation of $f$ if $f^{\prime}$ is homotopic to $f$, and we consider a collection of fixed points of a map $f$ unstable under perturbation if the collection vanishes under arbitrarily small perturbations of $f$.

Given any map $f: M \rightarrow M$ homotopic to $i d_{M}$ we are no longer able to construct the difference map as before, but we are able to construct the following analogous $\operatorname{map} \widetilde{\delta}: M \rightarrow \mathbb{R}$

$$
\widetilde{\delta}(x)=\text { the unique time } t \text { satisfying } \phi_{M}(t, x)=f(x)
$$

since $f(x)$ will be on the trajectory of $x$ and such a $t$ is uniquely determined when $M \neq S^{1}$. Unless otherwise stated, all maps $M \rightarrow M$ considered in this section are homotopic to $i d_{M}$.

Definition 3.1. A fixed point $p$ of $f: M \rightarrow M$ is proper if there is a sequence $\left\{t_{n}\right\} \subset \mathbb{R}$ satisfying:

(1) $t_{n} \rightarrow 0$

(2) $t_{2 n-1}<0$ and $t_{2 n}>0$ for $n=1,2, \ldots$ 
(3) $\operatorname{sign}\left(\widetilde{\delta}\left(\phi_{M}\left(t_{2 n-1}, p\right)\right) \cdot \widetilde{\delta}\left(\phi_{M}\left(t_{2 n}, p\right)\right)\right)=-1, n=1,2, \ldots$.

For example, $p \in \Sigma_{N}$ is proper when the graph of $\Delta_{p}$ intersects the horizontal axis transversely at $(0,0)$.

Theorem 3.2. If $p$ is a proper fixed point of $f$, then $p$ is a limit point of the set of fixed points of $f$.

Proof. The minimality of $\phi_{M}$ guarantees the existence of a sequence of real numbers $\left\{s_{i}\right\} \rightarrow \infty$ satisfying $p=\lim _{i}\left\{\phi_{M}\left(s_{i}, p\right)\right\}=\lim _{i}\left\{p_{i}\right\}$. Since $\phi_{M}$ is a continuous group action, for any $t$ we also have $\phi_{M}(t, p)=\lim _{i}\left\{\phi_{M}\left(t, p_{i}\right)\right\}$. Thus, for $n=$ $1,2, \ldots$ the continuity of $\widetilde{\delta}$ guarantees the existence of a corresponding $i_{n} \geq n$ with

$$
\operatorname{sign}\left(\widetilde{\delta}\left(\phi_{M}\left(t_{2 n-1}, p_{i_{n}}\right)\right) \cdot \widetilde{\delta}\left(\phi_{M}\left(t_{2 n}, p_{i_{n}}\right)\right)\right)=-1 .
$$

Then the arc

$$
A_{n} \stackrel{\text { def }}{=} \phi_{M}\left(\left[t_{2 n-1}, t_{2 n}\right], p_{i_{n}}\right)
$$

must contain a zero of $\widetilde{\delta}$ and thus a fixed point $q_{n}$ of $f$. Since the diameter of the $\operatorname{arcs} A_{n}$ goes to zero as $n \rightarrow \infty$ and since $\left\{p_{i_{n}}\right\}_{n} \rightarrow p, q_{n} \rightarrow p$.

Theorem 3.3. If $p$ is a fixed point of $f$ which is isolated from all other fixed points of $f$, then there is a neighborhood $U$ of $p$ and arbitrarily small perturbations $f_{\varepsilon}$ of $f$ which have no fixed points in $U$.

Proof. There is a local basis for $M$ at $p$ of the form

$$
\{Z \times[0,1] \mid Z \text { is zero-dimensional }\} \text { (see }[\mathrm{AM}] \text { ), }
$$

and since $p$ is isolated, there is such a basis element $Z_{0} \times[0,1]$ containing no fixed points other than $p$. With the exception of the arc containing $p$, the arcs in this basis element contain no fixed points, and so $\widetilde{\delta}$ will be of constant sign on each such arc. If in each neighborhood of $p$ consisting of arcs from $Z_{0} \times[0,1]$ there were $\operatorname{arcs}$ of different signs, there would be a sequence of "positive" and a sequence of "negative" arcs converging to the arc containing $p$. Then $\widetilde{\delta}$ would be constantly 0 on the arc containing $p$, contrary to the isolation of $p$. Hence, there must be a neighborhood $U$ of $p$ consisting of arcs on which $\widetilde{\delta}$ is either (a) non-negative or (b) non-positive, and without loss of generality we assume case (a). Let $\varepsilon(x): M \rightarrow[0, \varepsilon)$ be a map satisfying:

$$
\varepsilon(x)=\left\{\begin{array}{ll}
0 & \text { if } x \notin U \\
\text { positive } & \text { if } x=p
\end{array} .\right.
$$

Then the perturbed map $f_{\varepsilon}(x)=\phi_{M}(\widetilde{\delta}(x)+\varepsilon(x), x)$ contains no fixed point in $U$. In fact, the set of fixed points is unchanged but for the loss of $p$.

In much the same way as was done with a point, we could define an arc of fixed points to be proper when it is flanked by sequences converging to the endpoints, where the sign of $\widetilde{\delta}$ for terms in the sequences vary in the same way as above. It would then follow that there would be a sequence of fixed points not on the arc which converges to some point of the arc. If there is an isolated arc of fixed points, ideas similar to those above can be used to show that the entire arc is unstable under perturbation. Only the identity has $M$ as its fixed point set, and this vanishes under any time- $\varepsilon$ map of the flow. Since the only subcontinua of $M$ are points, arcs and 
$M$ itself, we are led to the conclusion that any isolated continuum of fixed points is unstable under perturbation.

It is important to realize that these results are special to maps homotopic to the identity. For example, $e$ is an isolated but stable fixed point of the shift automorphism and the involution $x \mapsto-x$ of the dyadic solenoid.

\section{Prime Solenoids Admit no Expansive Homeomorphism}

If each prime $p$ is a factor of only finitely many of the $n_{i}$ from the sequence $N=\left(n_{1}, n_{2}, \ldots\right)$, we refer to the corresponding solenoid $\Sigma_{N}$ as prime, and all other solenoids will be referred to as composite. Using the terminology of the introduction, it follows from $[\mathrm{K}]$ that $E_{N}$ is the entire group of homeomorphisms of a prime solenoid $\Sigma_{N}$. After we have demonstrated that no homeomorphism from $E_{N}$ (independent of $N$ ) can be expansive, it follows that no prime solenoid admits an expansive homeomorphism; that is, a homeomorphism $h$ for which there is a constant $c>0$ satisfying the condition that for all $x \neq y$ there is an $n \in \mathbb{Z}$ with

$$
d\left(h^{n}(x), h^{n}(y)\right)>c .
$$

This provides a partial answer to D. Bellamy's question of which solenoids admit an expansive homeomorphism. If $N=(n, n, \ldots)$, it follows from the proof of [W] that the shift automorphism on $\Sigma_{N}$ is expansive. All composite solenoids admit a shift-like automorphism; however, while this automorphism is continuum-wise expansive (see [Ka] for an explanation of this term), it is not generally expansive. To provide a complete characterization of the solenoids that admit expansive homeomorphisms, it remains to determine which composite solenoids admit expansive homeomorphisms.

Theorem 4.1. Independent of $N$, no $h \in E_{N}$ is expansive.

Proof. Given any two points $x, y$ in the same composant of $\Sigma_{N}$, the map $t \mapsto$ $x+\pi_{N}(t)$ provides a consistent orientation: $x<y$ if $x+\pi_{N}(t)=y$ for $t>0$ and $x>y$ if $x+\pi_{N}(t)=y$ for $t<0$. If a map $\Sigma_{N} \rightarrow \Sigma_{N}$ preserves this orientation, so will any iteration of this map; but if $\Sigma_{N} \rightarrow \Sigma_{N}$ reverses this orientation, any even-numbered iteration of this map will preserve orientation. Now we have the homeomorphism

$$
E_{N} \approx \Sigma_{N} \times G_{0}(N) \times \mathbb{Z}_{2}
$$

as described in $[\mathrm{K}]$, where $G_{0}(N)$ is the contractible subgroup consisting of all homeomorphisms $\left(\Sigma_{N}, e\right) \rightarrow\left(\Sigma_{N}, e\right)$ isotopic to $i d_{\Sigma_{N}}$ and $\Sigma_{N} \times\left\{i d_{\Sigma_{N}}\right\} \times\{0\}$ corresponds to translations. While the above homeomorphism $\approx$ does not respect the group structure of $E_{N}$, consideration of the above remarks on orientation shows that some amount of the structure of $\mathbb{Z}_{2}$ is reflected in the group of homeomorphisms: with $i d_{\Sigma_{N}} \sim\left(e, i d_{\Sigma_{N}}, 0\right)$ and $-i d_{\Sigma_{N}} \sim\left(e, i d_{\Sigma_{N}}, 1\right)$, any homeomorphism $h \sim(x, f, 0)$ composed with a homeomorphism of the same form will again be of the same form; while an even number of iterations of a homeomorphism $h \sim(x, f, 1)$ will be of the form $(y, g, 0)$.

Suppose then that $h \in E_{N}$ and that $h$ is expansive. Since $h \circ h$ is expansive when $h$ is (see, e.g., [Wa] Corollary 5.22.1), by the above considerations we may assume without loss of generality that $h$ is homotopic to translation by some $\xi \in$ $\Sigma_{N}$, from which it follows that for $n \in \mathbb{Z} h^{n}$ is homotopic to translation by $n \xi$. To reach a contradiction, we examine the consequences for the induced map 
$\widehat{h}: C\left(\Sigma_{N}\right) \rightarrow C\left(\Sigma_{N}\right)$ on the hyperspace of subcontinua $\Sigma_{N}$. Rogers showed in [R] that $C\left(\Sigma_{N}\right)$ is homeomorphic to the cone over $\Sigma_{N}$, Cone $\left(\Sigma_{N}\right)$. The proper nondegenerate subcontinua of $\Sigma_{N}$ are $\operatorname{arcs} A$ of the form $x+\pi_{N}(I)$ for some interval $I \subset \mathbb{R}$, and if one defines the length $\ell(A)$ to be the length of any such associated interval $I$, one sees that any two arcs of the same length are isometric since any two such arcs are translates of each other and the metric for $\Sigma_{N}$ is translation invariant. One can construct a homeomorphism $C\left(\Sigma_{N}\right) \approx C$ Cone $\left(\Sigma_{N}\right)$ as follows: the singletons are mapped to the bottom of the cone, all arcs of a given length are mapped to the same level of the cone, with the center of the arcs mapping to the corresponding point on the corresponding level of the cone, and $\Sigma_{N}$ is mapped to the top of the cone. Viewed differently, one can construct a Whitney function $w: C\left(\Sigma_{N}\right) \stackrel{\text { onto }}{\longrightarrow}[0,1]$ which has the same value on any two isometric subcontinua (see, e.g., [IN], p. 108). If an $\operatorname{arc} A \subset \Sigma_{N}$ is shorter than an $\operatorname{arc} B \subset \Sigma_{N}$, there is a translation $A+x \varsubsetneqq B$, and so $w(A)=w(A+x)<w(B)$, see [IN], p. 105. Thus, for proper non-degenerate subcontinua, $w$ is a function of length and any Whitney level $w^{-1}(t)$ for $t \in(0,1)$ is the solenoid $\Sigma_{N}(t)$ of arcs of the same length (see also $[\mathrm{KN}])$.

We proceed to show that for $f: \Sigma_{N} \rightarrow \Sigma_{N}$ homotopic to translation by $\gamma$, $\widehat{f}: C\left(\Sigma_{N}\right) \rightarrow C\left(\Sigma_{N}\right)$ "preserves the Whitney level on average." In much the same way as before, there is a map $\widetilde{\delta}: \Sigma_{N} \rightarrow \mathbb{R}$ lifting the map $f(x)-(x+\gamma)$. Fix a level $w^{-1}(t), t \in(0,1)$, with associated length $\lambda$ and define $d: \Sigma_{N} \rightarrow \mathbb{R}$ by

$$
d(x)=\widetilde{\delta}\left(x+\pi_{N}(\lambda)\right)-\widetilde{\delta}(x) .
$$

For a given $x$ and corresponding arc $A_{x}=x+\pi_{N}([0, \lambda])$, the value $d(x)$ is the difference $\ell\left(\widehat{f}\left(A_{x}\right)\right)-\ell\left(A_{x}\right)$. For each $x \in \Sigma_{N}$ the maps $\Delta_{x}$ and $\Delta_{x+\pi_{N}(\ell)}$ both have mean value $\delta_{0}=\int_{\Sigma_{N}} \widetilde{\delta}(x) d \mu_{N}$, and so $d$ has mean value 0 :

$$
\int_{\Sigma_{N}} d(x) d \mu_{N}=0
$$

As shown in Theorem 2.1, $d$ attains the value 0 uncountably many times. To each zero $x$ of $d$ the corresponding arc $A_{x} \in w^{-1}(t)$ satisfies $w\left(A_{x}\right)=w\left(\widehat{f}\left(A_{x}\right)\right)$.

By Corollary 3.4 of $[\mathrm{Ka}]$ there is a $\gamma>0$ so that one of the following holds for any given $\varepsilon>0$

(1) If $A \in C\left(\Sigma_{N}\right)$ and $0<\operatorname{diam}(A)<\gamma$, then there is a positive integer $n_{0}$ so that $\operatorname{diam}\left(h^{n}(A)\right)<\varepsilon$ for all $n \geq n_{0}$ or

(2) If $A \in C\left(\Sigma_{N}\right)$ and $0<\operatorname{diam}(A)<\gamma$, then there is a positive integer $n_{0}$ so that $\operatorname{diam}\left(h^{-n}(A)\right)<\varepsilon$ for all $n \geq n_{0}$.

Replacing $h$ with $h^{-1}$ if necessary, we assume without loss of generality that 1 holds. Choose

$$
\sigma<\min \left\{\gamma, \operatorname{diam}\left(\Sigma_{N}\right)\right\}, \text { let } \varepsilon=\sigma / 2 \text { and }
$$

let $K$ be the compact set $\left\{A \in C\left(\Sigma_{N}\right) \mid \operatorname{diam}(A) \leq \sigma\right\}$. Then $K=w^{-1}([0, t])$ for some $t \in(0,1)$ and $\left\{A \in C\left(\Sigma_{N}\right) \mid \operatorname{diam}(A) \leq \varepsilon\right\}=w^{-1}\left(\left[0, t^{\prime}\right]\right)$ for some $t^{\prime} \in(0, t)$. By 1 and the continuity of diamo $\hat{f}$ for any map $f: \Sigma_{N} \rightarrow \Sigma_{N}$ (see [IN]), for each $A \in K$ there is the least number $n_{A} \in\{1,2, \ldots\}$ with $\operatorname{diam}\left(h^{n_{A}}(B)\right)<\varepsilon$ for all $B$ in some neighborhood $U_{A}$ of $A$. By the compactness of $K$ there is a finite subcover of $\left\{U_{A} \mid A \in K\right\}$ :

$$
K \subset U_{A_{1}} \cup \cdots \cup U_{A_{m}} .
$$


THE DYNAMICS OF MAPS OF SOLENOIDS HOMOTOPIC TO THE IDENTITY

With $M=\max \left\{n_{A_{1}}, \ldots, n_{A_{m}}\right\}$, for all $A \in K$ there is an $n \in\{1,2, \ldots, M\}$ with $\operatorname{diam}\left(h^{n}(A)\right)<\varepsilon$. Since $\widehat{h^{n}}$ is a homeomorphism sending $\Sigma_{N} \mapsto \Sigma_{N}$ and since $\ell$ is continuous, the compactness of $K$ guarantees the existence of

$$
L=\max \left\{\ell\left(h^{n}(A)\right) \mid A \in K \text { and } n \in\{1,2, \ldots, M\}\right\} .
$$

Lemma 4.2. For all $A \in K$ and for all $n \in\{1,2, \ldots\}, \ell\left(h^{n}(A)\right) \leq L$.

Proof. Let $A \in K$ and $k \in\{1,2, \ldots\}$. Then $B=h^{n_{A}}(A) \in K$ and $\ell\left(h^{n}(A)\right) \leq L$ for all $n \leq n_{A}$ since $n_{A} \leq M$. Applying the same reasoning to $B$, we have that $h^{n_{A}+n_{B}}(A) \in K$ and $\ell\left(h^{n}(A)\right) \leq L$ for all $n \leq n_{A}+n_{B}$ and $n_{A}<n_{A}+n_{B}$. Proceeding in this manner a total of $k$ times, we can conclude $\ell\left(h^{n}(A)\right) \leq L$ for all $n \leq k$.

Let $\lambda$ and $\lambda^{\prime}$ be the lengths of arcs in $w^{-1}(t)$ and $w^{-1}\left(t^{\prime}\right)$ respectively and for $x \in \Sigma_{N}$ let $A_{x}=x+\pi_{N}([0, \lambda])$. For each $n \in\{1,2, \ldots\}$ let $F_{n}: \Sigma_{N} \rightarrow \mathbb{R}$ be the measurable function given by

$$
F_{n}\left(A_{x}\right)=\sup _{k \geq n}\left\{\ell\left(h^{k}\left(A_{x}\right)\right)\right\},
$$

which is well-defined by 1 . Then with $E_{n}=\left\{x \in \Sigma_{N} \mid F_{n}\left(A_{x}\right) \geq \lambda^{\prime}\right\}$, we have $E_{n+1} \subset E_{n}$ and $\cap E_{n}=\emptyset$, again by 1 . Then for the Haar measure $\mu_{N}$ we must have

$$
\lim _{n \rightarrow \infty} \mu_{N}\left(E_{n}\right)=0 .
$$

Choose $n_{0}$ so that $\mu_{N}\left(E_{n_{0}}\right)<\min \left\{\frac{\lambda-\lambda^{\prime}}{2 L}, \frac{1}{2}\right\}$. With $f=h^{n_{0}}$ (homotopic to a translation) we have the associated map

$$
d(x)=\widetilde{\delta}\left(x+\pi_{N}(\lambda)\right)-\widetilde{\delta}(x)
$$

and $\int_{\Sigma_{N}} d(x) d \mu_{N}=0$ as shown above. Notice that if $h^{n_{0}}\left(A_{x}\right) \in w^{-1}\left(\left[0, t^{\prime}\right]\right)$, $d(x) \leq \lambda^{\prime}-\lambda<0$ and so

$$
\begin{aligned}
\int_{\Sigma_{N}} d(x) d \mu_{N} & =\int_{E_{n_{0}}} d(x) d \mu_{N}+\int_{\Sigma_{N}-E_{n_{0}}} d(x) d \mu_{N} \\
& <L\left(\frac{\lambda-\lambda^{\prime}}{2 L}\right)+\left(\lambda^{\prime}-\lambda\right) \frac{1}{2}=0
\end{aligned}
$$

a contradiction. Hence, no such expansive $h$ can exist.

\section{REFERENCES}

[AM] J.M. Aarts and M. Martens, Flows on one-dimensional spaces, Fund. Math., 129 (1988), 39-58.

[B] H. Bohr, Almost periodic functions, Chelsea Pub. Co., New York, 1947.

[C1] A. Clark, Linear flows on $\kappa$-solenoids, Topology and its Applications, 94 (1999), 27-49.

[C2] A. Clark, Flows on solenoids are generically not almost periodic, Geometry and topology in dynamics, Contemp. Math., 246, Amer. Math. Soc., Providence, RI, 1999, 57-63.

[CHM] A. Chigogidze, K.H. Hofmann, J.R. Martin, Compact groups and fixed points, Trans. Amer. Math. Soc. 349, Number 11, (1997), 4537-4554.

[F] R.J. Fokkink, The Structure of Trajectories, Dissertation at the University of Delft, 1991.

[IN] A. Illanes and S.B. Nadler, Jr., Hyperspaces: Fundamentals and Recent Advances, Marcel Dekker, New York, Basel, 1999.

[Ka] H. Kato, The nonexistence of expansive homeomorphisms of a class of continua which contains all decomposable circle-like continua, Trans. A.M.S. 349, Number 9, (1997), 3645-3655. 
[K] J. Keesling, The group of homeomorphisms of a solenoid , Trans. Amer. Math. Soc. 172 (1972), 119-131.

[KN] J. Krasinkiewicz and S.B. Nadler, Jr., Whitney Properties, Fund. Math., 98 (1978), 165180.

[NS] V.V. Nemytskii and V.V. Stepanov, Qualitative Theory of Differential Equations, Princeton Univ. Press, Princeton, NJ, 1960.

[P] L. S. Pontryagin, Topological groups, Second Edition, Gordon and Breach, New York, 1966.

[Rob] C. Robinson, Dynamical systems: stability, symbolic dynamics, and chaos, CRC Press, Boca Raton, Ann Arbor, London, Tokyo, 1995.

[R] J.T. Rogers, Jr., Embedding the hyperspaces of circle-like plane continua, Proc. A. M. S. 29, (1971), 165-168.

[Wa] P. Walters, An introduction to ergodic theory, Springer Verlag, Berlin, Heidelberg, New York, 1982.

[W] R.F. Williams, A note on unstable homeomorphisms, Proc. A. M. S. 6 (1955), 308-309.

Department of Mathematics, University of North Texas, Denton, TX 76203-1430

E-mail address: alexc@unt.edu 